\title{
Practical Measurement of Absolute Velocity of Earth
}

\author{
Gurcharn S. Sandhu ${ }^{1}$ \\ ${ }^{1}$ Independent Researcher, India \\ Correspondence: Gurcharn S. Sandhu, \#48, Sector 61, Mohali, Punjab-160062, India. Tel: 91-981-462-2204. \\ E-mail: gurcharn.sandhu@gmail.com
}

Received: February 27, 2019

Accepted: March 24, 2019

Online Published: April 3, 2019

doi:10.5539/apr.v11n3p12

URL: http://dx.doi.org/10.5539/apr.v11n3p12

\begin{abstract}
In 1880, Albert Michelson conceived the idea of optically measuring the speed of earth through the solar system. However, the Michelson-Morley experiment conducted for detecting the expected motion of earth, gave null result. Einstein propounded his theory of Relativity on the assumption that speed of light is isotropic in ECI reference frame. The Relativity model can be invalidated through an unambiguous physical experiment that confirms anisotropy of light speed in ECI reference frame. Let us consider a $3 \mathrm{~km}$ long line segment AB, fixed on surface of earth. The proposed experiment for measuring absolute velocity of earth involves sending a picoseconds Laser pulse from location $A$ at time $T_{0}$ and detecting it at location $B$ at time $T_{1}$ to measure the time of flight $T_{a b}$ of the laser pulse from A to B. On the other hand, by sending a picoseconds Laser pulse from location B at time $T_{0}$ and detecting it at location $A$ at time $T_{2}$ we can measure the time of flight $T_{b a}$ from $B$ to $A$. For accurate measurement of times of flight $\mathrm{T}_{\mathrm{ab}}$ and $\mathrm{T}_{\mathrm{ba}}$ the precision atomic clocks located at $\mathrm{A}$ and $\mathrm{B}$ must be in absolute synchronization. The absolute velocity $U_{a b}$ will be given by $U_{a b} / c=\left(T_{a b}-T_{b a}\right) /\left(T_{a b}+T_{b a}\right)$. Final absolute velocity $U$ of earth can be computed from pulse flight timing record of 24 hours. For this we need to use the latest cutting-edge technology in Atomic Clocks, Pulsed Lasers, Ultrafast Detectors and Picosecond Time Interval Counters.
\end{abstract}

Keywords: absolute synchronization, absolute velocity, isotropy, physical space, pulsed laser, relativity

\section{Introduction}

It is well known that the orbital speed of earth is about $30 \mathrm{~km} / \mathrm{s}$. It is also known that our solar system moves in the Milky-Way Galaxy at about $200 \mathrm{~km} / \mathrm{s}$. It is estimated that the Milky-Way Galaxy itself moves at about $600 \mathrm{~km} / \mathrm{s}$ when measured with respect to a Cosmic Microwave Background (CMB) rest frame. If we define a 'Universal Reference Frame' as the one which is at rest with respect to the center of mass of the universe, then any motion with respect to such a Universal Reference Frame will be called 'absolute motion'. We may guess the absolute velocity of earth with respect to the Universal Reference Frame to be within a range of $400 \mathrm{~km} / \mathrm{s}$ to $800 \mathrm{~km} / \mathrm{s}$. But can we actually measure the absolute velocity of earth? As per the current Relativity dominated paradigm of fundamental Physics, there is no Universal Reference Frame, no absolute motion and hence no question of measuring the absolute velocity of earth. Well, in that case we can measure the absolute velocity of earth only after invalidating the Special Theory of Relativity which is founded on the assumed isotropy of light speed in earth centered inertial (ECI) reference frame. Or putting it other way round, if we somehow manage to practically measure the absolute velocity of earth, it will automatically invalidate the current paradigm of fundamental Physics. In this paper we introduce a novel technique for practically measuring the absolute velocity of earth.

In 1880, Albert A. Michelson conceived the idea of optically measuring the speed of earth through the solar system. For this purpose, he designed the "Michelson Interferometer" which proved to be of great value in many other experiments. He collaborated with colleague Edward Williams Morley, for improving the Michelson Interferometer and in 1887 the famous experiment was conducted (Michelson, 1887). Their experiment for the expected motion of the earth relative to the ether gave a null result. However, it has now been shown that the null result of the famous Michelson-Morley experiment (MMX) could be attributed to a conceptual flaw in its design (Sandhu, 2016). The most crucial step in the design of MMX that correlates the motion induced changes in the photon flight time in axial and transverse beams, to the corresponding changes in the phase of two beams is shown to be wrong and invalid. The null result of MMX inadvertently turned out to be the trigger for a paradigm shift in modern physics. Albert Einstein propounded his theory of Relativity which declared through a Postulate that the speed of light is isotropic in ECI reference frame as well as in all moving inertial reference frames. Thus, Relativity 
discarded the Newtonian notions of absolute space and time. Luckily, Einstein adopted the pre-existing concept of mass-energy equivalence and integrated it into the structure of Relativity. This mass-energy equivalence turned out to be the life-line of Relativity and sustained it against all odds (Sandhu, 2011).

\section{Absolute and Relativistic Clock Synchronization}

Since the time measured by all precision clocks is independent of their position or velocity in any reference frame, all clocks measure the Newtonian absolute time. However, to support his assumption of light speed isotropy in ECI frame, Albert Einstein introduced a different notion of time in his 1905 paper (Einstein, 1905). “...We have not defined a common 'time' for $A$ and B, for the latter cannot be defined at all unless we establish by definition that the 'time' required by light to travel from $A$ to $B$ equals the 'time' it requires to travel from $B$ to A". The "common time" thus defined in SR is not the absolute but relativistic time and this definition of 'time' formed the basis for relativistic synchronization convention or simply the "e-synchronization" of clocks. This arbitrary definition of relativistic time constitutes a fundamental departure from the Newtonian notion of absolute time. When the clocks at two spatially separated points A and B are adjusted or set to the relativistic time, it will ensure that the time interval measured for the light to travel from A to B will be equal to the time interval measured for the light to travel from B to A. In other words, if a group of identical clocks are adjusted or set to the relativistic time then the light speed measured with such clocks will always be isotropic. Reversing the argument, if precision time of a standard clock is distributed to a group of identical clocks through light (or EM) signals by assuming light speed isotropy in the ECI (or any other inertial) reference frame, then the time thus transferred to these clocks will be relativistic time and not the absolute time (Sandhu, 2013).

When the frequency and phase or displayed time of two or more precision atomic clocks are adjusted to match exactly in close-by position, the clocks are said to be in absolute synchronization. Since the time and frequency of any precision atomic clock is independent of their position in any reference frame, the absolute synchronization of two or more clocks will not be disturbed by any change in their position. Therefore, when two or more precision atomic clocks are in absolute synchronization, they will display absolute time, regardless of their state of motion with respect to any observer.

Since the speed c of light in vacuum is a property of physical space, it can be an isotropic constant only with respect to an absolute reference frame. Consider a line segment $\mathrm{AB}$ of length $\mathrm{D}$, moving in the absolute reference frame along $A B$. When a line segment $A B$ is moving with absolute velocity $U_{a b}$ along $A B$, a signal pulse will take longer to propagate from the trailing end $A$ to the leading end $B$ but will take shorter time from $B$ to $\mathrm{A}$. The time taken by a light pulse to propagate from point $\mathrm{A}$ to $\mathrm{B}\left(\mathrm{T}_{\mathrm{ab}}\right)$, is

$$
\mathrm{T}_{\mathrm{ab}}=\mathrm{D} /\left(\mathrm{c}-\mathrm{U}_{\mathrm{ab}}\right)
$$

Similarly, if $\mathrm{T}_{\mathrm{ba}}$ is the time taken by a pulse of light to propagate from point $\mathrm{B}$ to $\mathrm{A}$, then,

$$
\mathrm{T}_{\mathrm{ba}}=\mathrm{D} /\left(\mathrm{c}+\mathrm{U}_{\mathrm{ab}}\right)
$$

From Equations (1) and (2), it can be shown that,

$$
\mathrm{U}_{\mathrm{ab}} / \mathrm{c}=\left(\mathrm{T}_{\mathrm{ab}}-\mathrm{T}_{\mathrm{ba}}\right) /\left(\mathrm{T}_{\mathrm{ab}}+\mathrm{T}_{\mathrm{ba}}\right)
$$

Equation (3) shows that the absolute velocity $U_{a b}$ of two objects $A$ and $B$, can be determined by measuring the to and fro pulse propagation times between them (Sandhu, 2010). Essentially, the proposed experiment involves sending a picosecond Laser pulse from location $A$ at time $T_{0}$ and detecting it at location $B$ at time $T_{1}$ to measure the time of flight $T_{a b}$ of the light pulse from A to B. On the other hand, by sending a picosecond Laser pulse from location $B$ at time $T_{0}$ and detecting it at location $A$ at time $T_{2}$ we can measure the time of flight $T_{\text {ba }}$ of the light pulse from $B$ to A. For accurate measurement of times of flight $T_{a b}$ and $T_{b a}$ the precision atomic clocks located at $\mathrm{A}$ and $\mathrm{B}$ must be in absolute synchronization.

\section{Experimental Approach for any Line Segment}

\subsection{Test Setup}

The main experiment for measurement of Absolute Velocity of earth and our solar system is planned to be executed over a track length or line segment $\mathrm{AB}$ of about $3 \mathrm{~km}$. For this unique test, a level track with clear line of sight between test locations A and B, will be selected. Complete set of test instruments will consist of,
A. Two Cesium beam high performance Atomic Clocks (CAC), at an estimated cost of US $\$ 1,50,000$
B. Two Diode Pumped Solid State Pulsed LASERs with picoseconds pulse width and externally triggered single shot operation, at an estimated cost of US $\$ 45,000$
C. Two 3-channel, picosecond resolution Time Interval Counters (TIC), at an estimated cost of US $\$ 40,000$ 
D. Four ultrafast, InGaAs PIN, laser pulse detectors $\left(D_{1}, D_{2}\right)$ at an estimated cost of US $\$ 20,000$

E. Two Beam Splitter (BS), focusing optics and accessories at an estimated cost of US $\$ 7,000$

F. One Data Acquisition Computer (DAC) with application software, at an estimated cost of US $\$ 3,000$

To ensure that gravitational time dilation effects, if any, are completely avoided in this experiment, both test sites A and B must be located at the same gravitational potential. Out of the whole set of test instrumentation, performance of the two Cesium Atomic Clocks is most critical for satisfactory performance of the complete set. As such, we have to ensure the accuracy of these atomic clocks to be better than $\pm 1 \times 10^{-12}$ and stability to be better than $\pm 1 \times 10^{-13}$ under all environmental conditions. Frequency of these clocks must be adjustable with a resolution better than $1 \times 10^{-15}$ to ensure stable synchronization between the two clocks. In high performance Cesium Clocks, digital electronics continuously monitor environmental parameters and optimize the system performance to offset the effect of environmental conditions for ensuring long-term stability.

Further, each single set of test instruments will be positioned at opposite ends of the test line segment $\mathrm{AB}$ as shown in figure 1. Each set of test instruments will be mounted on a suitable trolley or mobile utility vehicle for convenience of shifting to the selected test location. The two selected test locations A and B must be interconnected with an efficient and smooth transportation facility for quick movement of test instruments from one location to another.

Each Laser beam will require a collimation optics at the BS end and focusing optics at the detector end. Two TICs from the two Test Sites A and B may be connected to the Data Acquisition Computer through an Internet Connection.

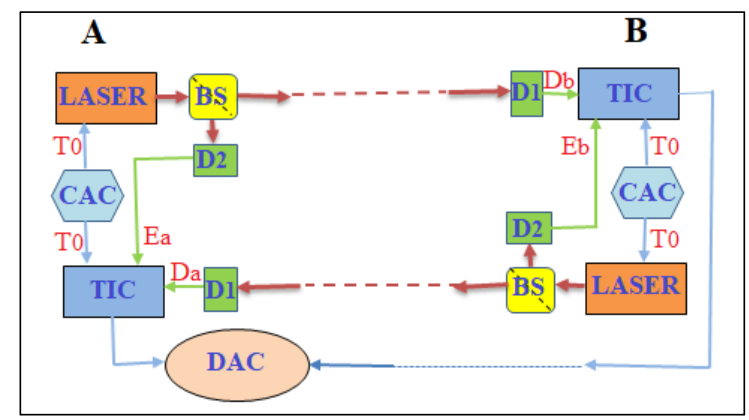

Figure 1. A full set of test instruments for a line segment AB

\subsection{Test Preparation}

In essence the absolute velocity measurement test consists of following basic steps.

(a) Bring the two atomic clocks at one location and synchronize their 1PPS signals in close-by position. This will constitute absolute synchronization of the two clocks. Then connect the 1PPS signals from two atomic clocks to the two input ports of one TIC to measure their clock offsets. Record these clock offsets for every 1PPS pulse from the atomic clocks for a period of two days. Study the pattern of these clock offset variations, estimate the level of frequency drift between two synchronized atomic clocks and carry out required frequency adjustments. Again synchronize the two atomic clocks and repeat the monitoring of their clock offsets for another two days. Study the pattern of residual clock offset variations and model these variations as function of time for making timing corrections during active data recording stage.

(b) Measure the pulse propagation delay times in each of the three input connecting lines for both TICs. These line delay times will be taken into account while computing various time intervals in both TICs. Position one set of test instruments at each test location and make necessary connections as shown in figure 1.

(c) Carry out the alignment of the laser heads from one location with the corresponding pulse detectors from the opposite location. We may use white light flash lamps for initial rough alignment. During such alignment, ultra-fast pulse detectors must be protected from damage from bright light and even day light.

(d) At each location, connect first 1 PPS output $T_{0}$ from the atomic clock to the trigger input of the pulsed laser and second 1PPS output to the zero-reference $\mathrm{T}_{0}$ input of the TIC. Connect the tapped laser pulse from the beam splitter (BS) to one of the pulse detectors $\left(\mathrm{D}_{2}\right)$ through fiber optic cable. Align the second pulse detector $\left(D_{1}\right)$ to the incoming laser pulse from the opposite location and connect the detected signal outputs from the two pulse detectors $\left(\mathrm{E}_{\mathrm{a}}, \mathrm{D}_{\mathrm{a}}\right.$ or $\left.\mathrm{E}_{\mathrm{b}}, \mathrm{D}_{\mathrm{b}}\right)$ to the two input ports of the respective TICs as shown in figure 1. 
(e) Connect the two measured time delay outputs of each TIC to the data acquisition computer. The measured time interval outputs of TIC(A) will be the emission time delay of the pulse $A$ to $B$, $\mathrm{T}_{\mathrm{Eab}}=\mathrm{E}_{\mathrm{a}}-\mathrm{T}_{0}$, and the detection time delay of the pulse $\mathrm{B}$ to $\mathrm{A}, \mathrm{T}_{\mathrm{Dba}}=\mathrm{D}_{\mathrm{a}}-\mathrm{T}_{0}$. Similarly, the measured time interval outputs of $\mathrm{TIC}(\mathrm{B})$ will be the emission time delay of the pulse $\mathrm{B}$ to $\mathrm{A}, \mathrm{T}_{\mathrm{Eba}}=\mathrm{E}_{\mathrm{b}}-\mathrm{T}_{0}$, and the detection time delay of the pulse $A$ to $B, T_{D a b}=D_{b}-T_{0}$.

The layout of the test instruments at the two test locations A and B is shown at figure 1. The ultrafast pulse detectors $\mathrm{D}_{1}$ may be fitted with suitable beam focusing optics or a telescope for ease of initial alignment of the Laser beams. The full set of test instruments must be calibrated for matching signal delays in various line components.

\subsection{Data Recording}

After completing the layout of the test setup as per figure 1, completing inter-connections between various test instruments, we are in a position to start recording the timing data from the two TICs into the Data Acquisition Computer. The synchronized 1PPS signal pulses from the two Atomic Clocks simultaneously trigger the emission of ultra-short Laser pulses from the opposite test locations and also feed the reference signals $\mathrm{T}_{0}$ to their respective TICs. Immediately after emission, the two Laser pulses pass through the Beam-splitters and the tapped-out pulse signals are fed into the detectors $\mathrm{D}_{2}$ through fiber-optic connections, from where the emission timing signals $E_{a}$ and $E_{b}$ are sent to the respective TICs. When the Laser pulses arriving from opposite locations are captured in the detectors $\mathrm{D}_{1}$, the detection timing signals $\mathrm{D}_{\mathrm{a}}$ and $\mathrm{D}_{\mathrm{b}}$ are sent to the respective TICs.

On receipt of detection timing signals, the TIC at location A will compute from the three timing inputs $\mathrm{T}_{0}, \mathrm{E}_{\mathrm{a}}$ and $\mathrm{D}_{\mathrm{a}}$, following two time-interval outputs and transfer them to the Data Acquisition Computer (DAC) for storage and processing.

And

Emission Time Delay at A, $\mathrm{T}_{\mathrm{Eab}}=\mathrm{E}_{\mathrm{a}}-\mathrm{T}_{0}$

Detection Time Delay at $\mathrm{A}, \quad \mathrm{T}_{\mathrm{Dba}}=\mathrm{D}_{\mathrm{a}}-\mathrm{T}_{0}$

Similarly, the TIC at location $\mathrm{B}$ will compute from the three timing inputs $\mathrm{T}_{0}, \mathrm{E}_{\mathrm{b}}$ and $\mathrm{D}_{\mathrm{b}}$, following two time-interval outputs and transfer them to the DAC for storage and processing.

$$
\text { Emission Time Delay at } \mathrm{B}, \quad \mathrm{T}_{\mathrm{Eba}}=\mathrm{E}_{\mathrm{b}}-\mathrm{T}_{0}
$$

And

$$
\text { Detection Time Delay at } \mathrm{B}, \quad \mathrm{T}_{\mathrm{Dab}}=\mathrm{D}_{\mathrm{b}}-\mathrm{T}_{0}
$$

After transferring the above time interval outputs to the DAC, the two TICs will get reset to the normal state, ready to receive the next $T_{0}$ reference signal. From this time interval data, the central DAC will compute the Laser pulse propagation time from location A to location $B\left(T_{a b}\right)$ and from location $B$ to location $A$ as under.

$$
\begin{array}{ll}
\text { Pulse propagation time from } \mathrm{A} \text { to } \mathrm{B}, & \mathrm{T}_{\mathrm{ab}}=\mathrm{T}_{\mathrm{Dab}}-\mathrm{T}_{\mathrm{Eab}} \\
\text { Pulse propagation time from } \mathrm{B} \text { to } \mathrm{A}, & \mathrm{T}_{\mathrm{ba}}=\mathrm{T}_{\mathrm{Dba}}-\mathrm{T}_{\mathrm{Eba}}
\end{array}
$$

Further, using equation (3), the central DAC will compute the component $\mathrm{U}_{\mathrm{ab}}$ of absolute velocity $\mathbf{U}$ of location A along the direction $\mathrm{AB}$ as,

$$
\mathbf{U}_{\mathbf{a b}}=\mathbf{c} \cdot \frac{\mathbf{T}_{\mathbf{a b}}-\mathbf{T}_{\mathbf{b a}}}{\mathbf{T}_{\mathbf{a b}}+\mathbf{T}_{\mathbf{b a}}}
$$

\begin{tabular}{|c|c|c|c|c|c|c|c|}
\hline 1PPS & $\mathrm{T}_{\text {Eab }}$ & $\mathrm{T}_{\mathrm{Dba}}$ & $\mathrm{T}_{\mathrm{Eba}}$ & $\mathrm{T}_{\mathrm{Dab}}$ & $\mathrm{T}_{\mathrm{ab}}$ & $\mathrm{T}_{\mathrm{ba}}$ & $\mathrm{U}_{\mathrm{ab}}$ \\
\hline Pulse No. & $\begin{array}{c}\text { Picosecond } \\
\text { Eqn.(4) }\end{array}$ & $\begin{array}{c}\text { Picosecond } \\
\text { Eqn.(5) }\end{array}$ & $\begin{array}{c}\text { Picosecond } \\
\text { Eqn.(6) }\end{array}$ & $\begin{array}{c}\text { Picosecond } \\
\text { Eqn.(7) }\end{array}$ & $\begin{array}{c}\text { Picosecond } \\
\text { Eqn.(8) }\end{array}$ & $\begin{array}{c}\text { Picosecond } \\
\text { Eqn.(9) }\end{array}$ & $\begin{array}{c}\mathrm{m} / \mathrm{s} \\
\text { Eqn.(10) }\end{array}$ \\
\hline 1 & $\mathrm{~T}_{\mathrm{Eab}}(1)$ & $\mathrm{T}_{\mathrm{Dba}}(1)$ & $\mathrm{T}_{\mathrm{Eba}}(1)$ & $\mathrm{T}_{\mathrm{Dab}}(1)$ & $\mathrm{T}_{\mathrm{ab}}(1)$ & $\mathrm{T}_{\mathrm{ba}}(1)$ & $\mathrm{U}_{\mathrm{ab}}(1)$ \\
\hline 2 & $\mathrm{~T}_{\mathrm{Eab}}(2)$ & $\mathrm{T}_{\mathrm{Dba}}(2)$ & $\mathrm{T}_{\mathrm{Eba}}(2)$ & $\mathrm{T}_{\mathrm{Dab}}(2)$ & $\mathrm{T}_{\mathrm{ab}}(2)$ & $\mathrm{T}_{\mathrm{ba}}(2)$ & $\mathrm{U}_{\mathrm{ab}}(2)$ \\
\hline . & . & $\cdot$ & . & $\cdot$ & 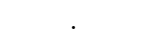 & & \\
\hline $\mathrm{n}$ & $\mathrm{T}_{\mathrm{Eab}}(\mathrm{n})$ & $\mathrm{T}_{\mathrm{Dba}}(\mathrm{n})$ & $\mathrm{T}_{\mathrm{Eba}}(\mathrm{n})$ & $\mathrm{T}_{\mathrm{Dab}}(\mathrm{n})$ & $\mathrm{T}_{\mathrm{ab}}(\mathrm{n})$ & $\mathrm{T}_{\mathrm{ba}}(\mathrm{n})$ & $\mathrm{U}_{\mathrm{ab}}(\mathrm{n})$ \\
\hline
\end{tabular}

The seven data elements, given by equations (4) to (10), will get computed every second and get stored in the DAC data sheets in tabulated form as represented in Table 1.

Table 1. Data Record for test line segment AB 
The test procedure described above yields a continuous record of absolute velocity component $U_{a b}$ along a test line segment $A B$, or of absolute velocity component $U_{a d}$ along a test line segment $A D$. Due to the rotation of earth, orientation of the line segment $\mathrm{AB}$ or $\mathrm{AD}$ changes cyclically with a period of one sidereal day. As such the continuous record of $\mathrm{U}_{\mathrm{ab}}$ or $\mathrm{U}_{\mathrm{ad}}$ will also show some periodic variations.

\subsection{Effect of Atmospheric Propagation Delays and Synchronization Errors}

The laser pulse propagation times $\mathrm{T}_{\mathrm{ab}}$ and $\mathrm{T}_{\mathrm{ba}}$ will be slightly increased due to atmospheric propagation delays in comparison to the propagation times under vacuum conditions. However, since the environmental conditions are same, the propagation times $T_{a b}$ and $T_{b a}$ both will be equally affected and hence their difference $\left(T_{a b}-T_{b a}\right)$ will remain unaffected. Therefore, the absolute velocity computed with equation (10) will remain unaffected by atmospheric propagation delays and environmental conditions. This is a major benefit of the proposed technique for practical measurement of absolute velocity of earth.

To minimize synchronization errors between two identical Cesium beam atomic clocks, we need to first study the variation of their clock offsets over a period of five to six days and eliminate their estimated frequency offsets through necessary frequency adjustments. Further, during active test recording phase too we may conduct synchronization checks periodically, say at every six hourly intervals to have a quantitative estimate of residual synchronization errors. For this we may either bring the two clocks together at one location and check their clock offsets with a TIC or we may use a third Cesium clock and TIC as a portable system to compare the clock offsets of the two atomic clocks at the test locations.

At any instant of time during the test data recording, let the estimated synchronization error between the two test clocks be $T_{\mathrm{SE}}$, such that clock $\mathrm{B}$ is lagging behind clock $\mathrm{A}$ by $\mathrm{T}_{\mathrm{SE}}$. That is, the 1PPS pulse from clock $\mathrm{B}$ will be delayed from the 1 PPS pulse of clock $A$ by $T_{S E}$, or $T_{0}(A)$ will lead $T_{0}(B)$ by a time interval of $T_{\text {SE }}$. Therefore, equation (8) will get modified (Sandhu, 2010) to,

Synchronization Error corrected Pulse propagation time from A to $B, T_{a b}^{\prime}=T_{D a b}-T_{E a b}+T_{S E}$

And equation (9) will get modified to,

Synchronization Error corrected Pulse propagation time from B to A, $\mathrm{T}_{\mathrm{ba}}^{\prime}=\mathrm{T}_{\mathrm{Dba}}-\mathrm{T}_{\mathrm{Eba}}-\mathrm{T}_{\mathrm{SE}}$

Hence, after correcting for the estimated Synchronization Error $\mathrm{T}_{\mathrm{SE}}$ equation (10) will get modified to,

$$
\mathbf{U}_{\mathrm{ab}}=\mathbf{c} \cdot \frac{\left(\mathrm{T}_{\mathbf{a b}}-\mathrm{T}_{\mathbf{b a}}\right)+2 \cdot T_{S E}}{\mathbf{T}_{\mathbf{a b}}+\mathrm{T}_{\mathrm{ba}}}
$$

Equation (10A) represents a timing correction in final computation of absolute velocity that accounts for a constant or steadily varying synchronization error $\mathrm{T}_{\mathrm{SE}}$ between periodic synchronization checks.

\section{Computation of Absolute Velocity Vector $U$ from Test Data}

\subsection{Rotation of Test Line Segments in Fixed Reference Coordinate System XYZ}

Let $\mathrm{L}$ be the latitude and $\mathrm{L}_{\mathrm{ng}}$ be the longitude of point $\mathrm{A}$ of the test line segment. Let us refer the line segment $\mathrm{AB}$ and the absolute velocity vector $\mathbf{U}$ to a fixed Coordinate system $\mathrm{XYZ}$ with its origin at center $\mathrm{P}$ of the Latitude circle and $\mathrm{Z}$ axis along the rotational axis of earth, pointing towards North Celestial Pole (Figure 2. (a)). Thus, the $\mathrm{XY}$ plane will be parallel to the earth's equatorial plane. Let us locate the XY plane on the latitude circle and fix the direction of $\mathrm{X}$-axis parallel to the line joining the center of earth to the Vernal Equinox on the Celestial Equator. Then the direction of Y-axis will get fixed by right hand rule (Figure 2. (b)). Let the absolute velocity vector $\mathbf{U}$ make an angle $\delta$ with the polar Z-axis and let the meridian plane containing the $\mathbf{U}$ vector make an angle $\alpha$ with the $\mathrm{XZ}$ meridian plane. That is, let Right Ascension (RA) of the $\mathrm{U}$ vector be $\alpha$ and Declination (Dec) be $\left(90^{\circ}-\delta\right)$. The horizon plane at point $\mathrm{A}$ will contain the line segments $\mathrm{AB}, \mathrm{AC}$ and $\mathrm{AD}$ (Figure 2. (c)). We need to compute the direction cosines of line segments $\mathrm{AB}, \mathrm{AC} \& \mathrm{AD}$, and the $\mathrm{U}$ vector for determining the component of $\mathrm{U}$ along different line segments.

As the earth rotates about its axis, the radial arm PA will rotate in XY plane, about Z-axis. At any instant of time $t$ (sidereal time) let the radial arm PA make an angle $\phi$ with the $\mathrm{X}$-axis. We can now compute the direction cosines (DCs) of line segments $\mathrm{AB}, \mathrm{AC}$ and $\mathrm{AD}$ as a function of rotation angle $\phi$ or time $t$. Thus, the angle $\theta_{\mathrm{u}}$ between test line segment $\mathrm{AB}$ or $\mathrm{AD}$ and the $\mathbf{U}$ vector can be computed from instant to instant. During the course of one day, $\phi$ varies from 0 to $360^{\circ}$. In the Horizon plane at A, local west to east direction is parallel to the local tangent to the latitude circle. Therefore, when the line segment $\mathrm{AB}$ is aligned west to east, its DCs can be obtained from the DCs of radial arm PA which makes an angle $\phi$ with the $\mathrm{X}$-axis. The line segment AC is aligned with the local south to north direction at $\mathrm{A}$ and is contained in the meridian plane passing through point $\mathrm{A}$. When a meridian circle cuts 
the latitude circle at point $\mathrm{A}$, line segment $\mathrm{AC}$ is tangent to the meridian circle at $\mathrm{A}$ and hence perpendicular to the central radial arm OA. Thus, the DCs of line segment AC can be obtained from the DCs of the central radial arm $\mathrm{OA}$ which is inclined to the equatorial plane at an angle $\mathrm{L}$. When a line segment $\mathrm{AD}$, lying in the Horizon plane, is inclined to the local W-E direction at an angle $\beta$, it can be first resolved into two perpendicular components along $\mathrm{AB}$ and $\mathrm{AC}$ for the purpose of computing its $\mathrm{DCs}$.

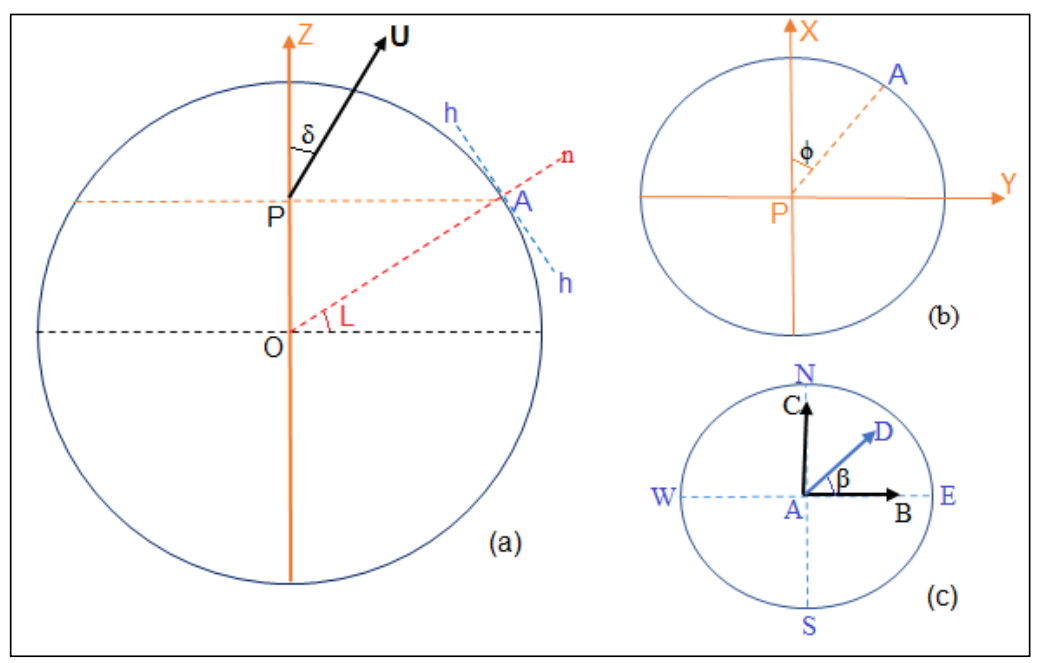

Figure 2. (a) $\mathrm{OA}$ is radius of earth and $\mathrm{L}$ the latitude of $\mathrm{A}$. PA is the latitude radius. $\mathrm{OZ}$ is the rotation axis of earth. $\mathrm{PZ}$ is Z-axis of the fixed XYZ coordinate system centered at P. A-n is the local vertical at A and $h-h$ is the local Horizon plane perpendicular to A-n. (b) Section at the Latitude circle. PA rotates in fixed X-Y coordinate plane. (c) Line segments in the Horizon Plane h-h with W-E tangent to the Latitude circle

\subsection{Direction Cosines of Velocity Vector $U$ and Line Segment AD}

Let us first compute the DCs of $\mathbf{U}$ vector which are independent of angle $\phi$ and time t. These DCs only depend on angles $\alpha$ and $\delta$ as follows.

$$
\mathrm{DC}_{\mathrm{U}}=\left[1_{\mathrm{u}}, \mathrm{m}_{\mathrm{u}}, \mathrm{n}_{\mathrm{u}}\right]=[\sin (\delta) \cdot \cos (\alpha), \sin (\delta) \cdot \sin (\alpha), \cos (\delta)]
$$

Since PA makes an angle $\phi$ with $\mathrm{X}$-axis, its tangent line $\mathrm{AB}$ makes an angle $\phi$ with $\mathrm{Y}$-axis.

$$
\text { DCs of } \mathrm{AB}=[-\sin (\phi), \cos (\phi), 0]
$$

Line segment $\mathrm{AC}$, being perpendicular to $\mathrm{OA}$, makes an angle $\mathrm{L}$ with $\mathrm{Z}$-axis and the meridian plane containing $\mathrm{AC}$ makes an angle $\phi$ with $\mathrm{XZ}$ plane. Therefore, DCs of AC will be,

$$
\text { DCs of } A C=[-\sin (L) \cdot \cos (\phi),-\sin (L) \cdot \sin (\phi), \cos (L)]
$$

Since the line segment $\mathrm{AD}$ makes an angle $\beta$ with local East direction, its components along $\mathrm{AB}$ and $\mathrm{AC}$ are $\mathrm{AD}$ $\cos (\beta)$ and $A D \sin (\beta)$ respectively. Therefore,

$$
\text { DCs of } A D=[-\cos (\beta) \cdot \sin (\phi)-\sin (\beta) \cdot \sin (L) \cdot \cos (\phi), \cos (\beta) \cdot \cos (\phi)-\sin (\beta) \cdot \sin (L) \cdot \sin (\phi), \sin (\beta) \cdot \cos (L)]
$$

Or,

$$
\begin{aligned}
& \mathrm{l}_{\mathrm{AD}}=-\{\cos (\beta) \cdot \sin (\phi)+\sin (\beta) \cdot \sin (\mathrm{L}) \cdot \cos (\phi)\} \\
& \mathrm{m}_{\mathrm{AD}}=\{\cos (\beta) \cdot \cos (\phi)-\sin (\beta) \cdot \sin (\mathrm{L}) \cdot \sin (\phi)\} \\
& \mathrm{n}_{\mathrm{AD}}=\{\sin (\beta) \cdot \cos (\mathrm{L})\}
\end{aligned}
$$

If $\theta_{\mathrm{u}}$ is the angle between vector $\mathbf{U}$ and the line segment $\mathrm{AD}$, then $\cos \left(\theta_{\mathrm{u}}\right)$ will be given by the dot product of DCs of $\mathrm{U}$ and $\mathrm{AD}$ from equations (11) and (14).

$$
\begin{aligned}
\cos \left(\theta_{\mathrm{u}}\right)= & \sin (\delta) \cdot \cos (\alpha) \cdot 1_{\mathrm{AD}}+\sin (\delta) \cdot \sin (\alpha) \cdot \mathrm{m}_{\mathrm{AD}}+\cos (\delta) \cdot \mathrm{n}_{\mathrm{AD}} \\
=- & \sin (\delta) \cdot \cos (\alpha) \cdot\{\cos (\beta) \cdot \sin (\phi)+\sin (\beta) \cdot \sin (\mathrm{L}) \cdot \cos (\phi)\} \\
& \quad+\sin (\delta) \cdot \sin (\alpha) \cdot\{\cos (\beta) \cdot \cos (\phi)-\sin (\beta) \cdot \sin (\mathrm{L}) \cdot \sin (\phi)\}
\end{aligned}
$$




$$
\begin{aligned}
+\cos (\delta) \cdot\{\sin (\beta) \cdot \cos (L)\} \\
=\cos (\delta) \cdot \sin (\beta) \cdot \cos (L)-\sin (\delta) \cdot\{\cos (\phi) \cdot[\cos (\alpha) \cdot \sin (\beta) \cdot \sin (L)-\sin (\alpha) \cdot \cos (\beta)]+ \\
\quad \sin (\phi) \cdot[\cos (\alpha) \cdot \cos (\beta)+\sin (\alpha) \cdot \sin (\beta) \cdot \sin (L)]\}
\end{aligned}
$$

During the course of one day, $\phi$ varies from 0 to $360^{\circ}$. Therefore, the magnitude of $\cos \left(\theta_{\mathrm{u}}\right)$ will vary sinusoidally during one sidereal day with some constant shift of the mean value from zero. Constant shift in $U_{A D}=U \cdot \cos \left(\theta_{u}\right)$ is given by $U \cdot \cos (\delta) \cdot \sin (\beta) \cdot \cos \left(\mathrm{L}\right.$. From the recorded data of $\mathrm{U}_{\mathrm{AD}}$ for one sidereal day, the 'mean value' $\mathrm{M}$ in $\mathrm{m} / \mathrm{s}$ will correspond to mean of $\mathrm{U} \cdot \cos \left(\theta_{\mathrm{u}}\right)$ given by equation (15) (for $\beta>0$ ),

$$
\begin{array}{cc} 
& \mathrm{M}=\mathrm{U} \cdot \cos (\delta) \cdot \sin (\beta) \cdot \cos (\mathrm{L}) \\
\text { Or } & \mathrm{U} \cdot \cos (\delta)=\mathrm{M} /[\sin (\beta) \cdot \cos (\mathrm{L})]
\end{array}
$$

\subsection{Diurnal Variation of Velocity Component $U_{a d}$}

The magnitude of $\cos \left(\theta_{\mathrm{u}}\right)$ will vary sinusoidally during one sidereal day with some constant shift of the mean value from zero. Therefore, experimentally determined absolute velocity $\mathrm{U}_{\mathrm{AD}}$ along the line segment $\mathrm{AD}$ will be plotted on $\mathrm{Y}$-axis while instant to instant varying values of $\phi(t)$ will be plotted along $\mathrm{X}$-axis. From equation (15), velocity term $\mathrm{U}_{\mathrm{ad}}$ can be expressed as a single sinusoidal term superposed over a constant term $[\cos (\delta) \cdot \sin (\beta) \cdot \cos (\mathrm{L})]$ as,

$$
\mathrm{Uad}=\mathrm{U} \cdot \cos \left(\theta_{\mathrm{u}}\right)=\mathrm{U} \cdot[\cos (\delta) \cdot \sin (\beta) \cdot \cos (\mathrm{L})]-\mathrm{U} \cdot \sin (\delta) \cdot \mathrm{Ab} \cos (\phi-\mathrm{b})
$$

Where

$$
A_{b}=\sqrt{1-(\sin (\beta) \cdot \cos (L))^{2}}
$$

And the derivative of $U_{a d}$ with respect to $\phi$, giving slope of the curve, is given by,

$$
\mathrm{d}\left(\mathrm{U}_{\mathrm{ad}}\right) / \mathrm{d} \phi=\mathrm{U} \cdot \sin (\delta) . \mathrm{A}_{\mathrm{b}} \sin (\phi-b)
$$

From equations (15) and (17) the phase offset parameter $b$ is given by,

$$
\tan (b)=\frac{\cos (\alpha) \cdot \cos (\beta)+\sin (\alpha) \cdot \sin (\beta) \cdot \sin (L)}{\cos (\alpha) \cdot \sin (\beta) \cdot \sin (L)-\sin (\alpha) \cdot \cos (\beta)}
$$

Or

$$
\frac{\sin (b)}{\cos (b)}=\frac{\cos (\beta)+\tan (\alpha) \cdot \sin (\beta) \cdot \sin (L)}{\sin (\beta) \cdot \sin (L)-\tan (\alpha) \cdot \cos (\beta)}
$$

The $U_{\text {ad }}$ curve of equation (17) makes a 'mean value' crossing, with positive slope, when the sinusoidal term $\cos (\phi-b)$ is zero and the $\sin (\phi-b)$ term in equation (17A) is positive. If the instantaneous value of $\phi$ is $\phi_{0}$ at this 'mean value' crossing then,

Or

$$
\begin{aligned}
& \phi_{0}-\mathrm{b}=\pi / 2 \\
& \mathrm{~b}=\phi_{0}-\pi / 2
\end{aligned}
$$

Thus, phase offset parameter $\mathrm{b}$ can be determined from the mean cross-over point, with positive slope, in the experimental data curve of $U_{a d}$. From equation (19) we can correlate $b$ with $\alpha$, the RA of absolute velocity vector $\mathbf{U}$ as,

Or

$$
\tan (\alpha) \cdot[\cos (b) \cdot \sin (\beta) \cdot \sin (L)+\sin (b) \cdot \cos (\beta)]=[\sin (b) \cdot \sin (\beta) \cdot \sin (L)-\cos (b) \cdot \cos (\beta)]
$$

$$
\tan (\alpha)=\frac{\sin (b) \cdot \sin (\beta) \cdot \sin (L)-\cos (\beta) \cdot \cos (b)}{\cos (b) \cdot \sin (\beta) \cdot \sin (L)+\sin (b) \cdot \cos (\beta)}
$$

Let $A_{m}$ be the amplitude of sinusoidal variation of experimental $U_{a d}$ curve represented by equation (17). Then $A_{m}$ will be half of the difference between maximum and minimum values of $U_{a d}$ recorded during one sidereal day. Therefore, from equation (17),

$$
\text { U. } \sin (\delta)=A_{m} / A_{b}
$$

Squaring and adding equations (16) and (22) we get,

$$
\mathrm{U}^{2}=\left(\mathrm{A}_{\mathrm{m}} / \mathrm{A}_{\mathrm{b}}\right)^{2}+\mathrm{M}^{2} /\left[\sin ^{2}(\beta) \cdot \cos ^{2}(\mathrm{~L})\right]
$$


Or

$$
\begin{gathered}
U=\sqrt{\left(A_{m} / A_{b}\right)^{2}+M^{2} /\left[\sin ^{2}(\beta) \cdot \cos ^{2}(L)\right]} \\
\tan (\delta)=\frac{A_{m} \cdot \sin (\beta) \cdot \cos (L)}{A_{b} \cdot M}
\end{gathered}
$$

With this we obtain all three parameters of the absolute velocity vector $\mathbf{U}$, namely the magnitude $\mathrm{U}$ is obtained from equation (24), angle $\delta$ (i.e. $\pi / 2-\mathrm{Dec}$ ) is obtained from equation (25) and RA angle $\alpha$ is obtained from equations (20) and (21).

\subsection{Correlation between angle $\phi$, local sidereal time and local standard time}

In the foregoing analysis angle $\phi$ is a measure of angular displacement of the local meridian of $A$ from the reference plane XZ. Since X-axis is aligned in the direction of vernal equinox, angle $\phi$ represents the angular displacement of radial line PA from PX in the XY plane. On the other hand, the angular displacement of local meridian of A from the fixed direction of vernal equinox can be expressed as the Hour Angle of the vernal equinox or the Local Sidereal Time (LST) of test site A.

The Local Sidereal Time $($ LST) $=$ Hour Angle of the vernal equinox.

Or,

$$
\text { LST }=\text { RA of the Sun on current date }+ \text { Hour Angle (HA) of the Sun }
$$

During apparent motion of the sun on the celestial sphere the sun appears to cross the vernal equinox on 21-22 March every year. So, the Right Ascension (RA) of the sun is zero on 21-22 March. Thereafter the RA of sun increases by approximately $1^{\circ}$ per day or $360^{\circ}$ in full year. Therefore, for computing RA of sun on any other date after 22 March, we need to first compute the number of days elapsed from 22 March to the given date as $\lambda_{\mathrm{d}}$. Then convert these number of days to $\lambda^{0}$ as RA of sun.

$$
\lambda^{\mathrm{o}}=\lambda_{\mathrm{d}} \times 360 / 365
$$

At Greenwich (Longitude $=00$ ) on 22 March at 12:00 hrs UTC, RA(sun) $=0$ and HA(sun) $=0$.

Therefore, Greenwich True Sidereal Time (GTST) at 12:00 UTC on 22 March $=0+0=0$

And GTST @ $12.00 \mathrm{UTC}$ on current date $(\mathrm{CD})=[\mathrm{RA}(\text { sun })]_{\mathrm{cd}}+0=\left[\lambda^{\circ}\right]_{\mathrm{cd}}+0$

GTST @ $\mathrm{T}_{\text {utc }}$ (Hours in decimals) on current date $=\left[\lambda^{\circ}\right]_{\mathrm{cd}}+\left(\mathrm{T}_{\text {utc }}-12.00\right) \times 360 / 23.93447$

If $\mathrm{L}_{\mathrm{ng}}$ is the local longitude (in degrees) of the test site $\mathrm{A}$, then

$$
\begin{aligned}
\text { LST } @ \mathrm{~T}_{\text {utc }} \text { (Hours in decimals) on current date } & =\mathrm{GTST} @ \mathrm{~T}_{\text {utc }} \text { on current date }+\mathrm{L}_{\mathrm{ng}} \\
& =\left[\lambda^{\circ}\right]_{\mathrm{cd}}+\left(\mathrm{T}_{\text {utc }}-12.00\right) \times 360 / 23.93447+\mathrm{L}_{\mathrm{ng}}
\end{aligned}
$$

Or LST $\mathrm{CD} @ \mathrm{~T}_{\text {utc }}=(\pi / 180) .\left[\lambda^{\circ}\right]_{\mathrm{cd}}+2 \pi .(11.93447) / 23.93447+\mathrm{L}_{\mathrm{ng}} \times(\pi / 180)+2 \pi . \mathrm{T}_{\text {utc }} / 23.93447 \quad$ radians

Or $\operatorname{LST}_{\mathrm{CD}} @ \mathrm{~T}_{\text {utc }}=(\pi / 180) .\left[\lambda^{0}\right]_{\mathrm{cd}}+2 \pi .(11.93447) / 23.93447+\mathrm{L}_{\mathrm{ng}} \times(\pi / 180)+2 \pi . \mathrm{T}_{\text {utc }} / 86164 \quad$ radians, where $\mathrm{T}_{\text {utc }}$ is in seconds.

Or

$\phi \quad$ in radians $=(\pi / 180) .\left[\lambda^{0}\right]_{\text {cd }}+2 \pi .(11.93447) / 23.93447+\mathrm{L}_{\mathrm{ng}} \times(\pi / 180)+2 \pi . \mathrm{T}_{\text {utc }} / 86164$

Or

$$
\left.\phi(t)=K_{d} \text { (in radians }\right)+(2 \pi / 86164) . T_{\text {utc }} \quad \text { where } T_{\text {utc }} \text { is in seconds }
$$

Such that

$$
\mathrm{K}_{\mathrm{d}}(\text { in radians })=(\pi / 180) \cdot\left[\lambda^{0}\right]_{\mathrm{cd}}+2 \pi \cdot(11.93447) / 23.93447+\mathrm{L}_{\mathrm{ng}} \times(\pi / 180)
$$

Therefore, experimentally determined absolute velocity $\mathrm{U}_{\mathrm{ad}}$ along the line segment $\mathrm{AD}$ will be plotted on $\mathrm{Y}$-axis while instant to instant varying values of $\phi(t)$ given by equations (31) and (32) will be plotted along $X$-axis. The sinusoidally varying $U_{\text {ad }}$ vs $\phi(t)$ plot for a period of one day or more will yield all parameters of the absolute velocity vector $\mathbf{U}$.

Since $U_{a d}$ is directly proportional to $\left(T_{a d}-T_{d a}\right.$ ) from equation (10), a typical plot of recorded timing difference $\left(\mathrm{T}_{\mathrm{ad}}-\mathrm{T}_{\mathrm{da}}\right.$ ) Vs. rotation angle $\phi$ is shown in figure 3. $\mathrm{X}_{1}$ and $\mathrm{X}_{2}$ represent the instants when the (site A) local meridian points in the direction of Vernal Equinox. Thus, from $X_{1}$ to $X_{2}$ rotation angle $\phi$ changes by $2 \pi$ radians and the curve between $\mathrm{X}_{1}$ and $\mathrm{X}_{2}$ represents the data record of one sidereal day. Such timing data records for 
subsequent days of testing will be identical to this plotted data between $\mathrm{X}_{1}$ to $\mathrm{X}_{2}$ except for small random fluctuations due to the clock synchronization instability errors. Therefore, if we superpose 5 consecutive days test record data between $\mathrm{X}_{1}$ and $\mathrm{X}_{2}$ and obtain their instant to instant average plot, such random fluctuations in test data can be minimized.

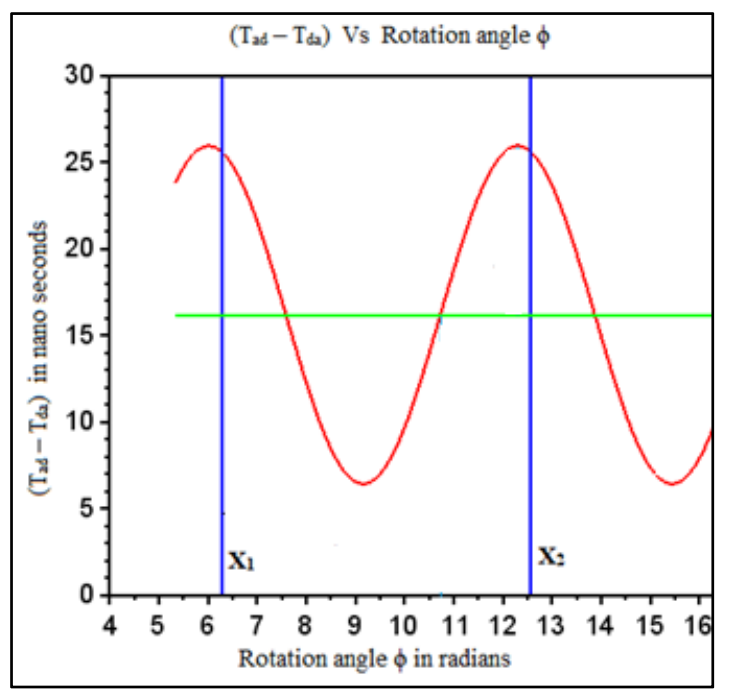

Figure 3. A typical plot of recorded timing difference $\left(\mathrm{T}_{\mathrm{ad}}-\mathrm{T}_{\mathrm{da}}\right)$ Vs. rotation angle $\phi$

\section{Tentative Time and Cost Estimates for the Project}

The subject experimental project, for measurement of Absolute Velocity of earth, is expected to be completed within 6 months after the provision of funds. Total cost of the project is estimated to be within US $\$ 3,20,000$ which includes $\$ 2,65,000$ for Test Instrumentation and $\$ 55,000$ for Administrative Overheads. The infrastructure and admin facilities are expected to be utilized from the parent Academic Institute or Research Establishment. Apart from the advanced cutting-edge technology involved in required Cesium Atomic Clocks, DPSS Pulsed Lasers, Ultrafast Pulse Detectors and Picosecond resolution Time Interval Counters, the actual conduct of the experiment and development of associated computational software, is fairly simple and within the capability of most Academic Institutes and Research Establishments.

\section{Possible Repercussions of the Test}

Practical measurement of absolute velocity of earth will validate Newtonian notions of absolute space, absolute time and absolute motion. It will invalidate Einstein's Theory of Relativity and junk the notions of motion-induced length contraction and time dilation. The Relativity theories, both Special and General (Einstein, 1916), had a tremendous sway over the minds of many generations of Physicists and they are treated as main pillars of Modern Physics (Sandhu, 2011). Hence the invalidation of Relativity with the measurement of absolute velocity of earth will surely provide a major stimulus to the mainstream community of Physicists and trigger a paradigm shift in Fundamental Physics.

It will re-establish the validity and utility of absolute clock synchronization and facilitate international Time keeping Labs to transfer absolute time by taking into account the absolute velocity of earth. Thus, Coordinated Universal Time (UTC) and International Atomic Time (TAI) will get hooked on to the absolute time in place of currently maintained Relativistic time (Sandhu, 2012). It will also facilitate putting precision Atomic Clocks in spaceships for enhanced navigation in deep space flights. Currently, the relativistic time cannot be maintained through e-synchronization process in space ships moving at high speeds and located far away from the earth.

Validation of Newtonian absolute space will revive fundamental research in physical space or aether. The notion of 4-D spacetime continuum as a physical entity will get invalidated leading to a fresh search for a valid theory of gravitation. It will lead scientific community to finally abandon some of the prominent cosmological models like Big Bang, Black Holes, (Sandhu, 2017) expanding universe etc. and begin a fresh search for the origin of matter particles and fields. 


\section{References}

Einstein, A. (1905). On the electrodynamics of moving bodies. Annalen der Physik., 17, 891.

Einstein, A. (1916). Relativity: The Special and General Theory. bartleby.com, New York-2000, 80.

Michelson, A. A., \& Morley, E. W. (1887). On the Relative Motion of the Earth and the Luminiferous Ether. American Journal of Science, 34, 333-345. http://dx.doi.org/10.2475/ajs.s3-34.203.333

Sandhu, G. S. (2010). Proposed experiment for detection of absolute motion. Physics Essays, 23(3), 442-450. http://dx.doi.org/10.4006/1.3454849

Sandhu, G. S. (2011). Relativity: a pillar of modern physics or a stumbling block. Proc. of SPIE,. 8121, 812109. http://dx.doi.org/10.1117/12.904607

Sandhu, G. S. (2012). Detection of absolute motion through measurement of synchronization offsets. Applied Physics Research, 4(4), 73-82. http://dx.doi.org/10.5539/apr.v4n4p73

Sandhu, G. S. (2013). Erroneous UTC Maintained by Timing Labs due to Mix-Up between Relativistic and Absolute Time. Applied Physics Research, 5(6), 83-92. http://dx.doi.org/10.5539/apr.v5n6p83

Sandhu, G. S. (2016). Fundamental Invalidity of all Michelson-Morley type Experiments. Applied Physics Research; Vol. 8, No. 3 (2016). http://dx.doi.org/10.5539/apr.v8n3p45

Sandhu G. S. (2017). Distinct doppler effects for spontaneously emitted photons and continuously emitted waves. Applied Physics Research, 9(4). http://dx.doi.org/10.5539/apr.v9n4p44

\section{APPENDIX}

\section{Details of Test Instrumentation}

In a practical experiment to test Light Speed anisotropy in Earth Centered Inertial (ECI) reference frame Laser Pulses are exchanged between two test sites, A and B separated by a distance of about $3 \mathrm{~km}$. and their flight times are recorded with sophisticated instrumentation.

\section{Set of Instruments}

A set of instruments used at one site (A) along with their schematic connections is shown at figure 4.

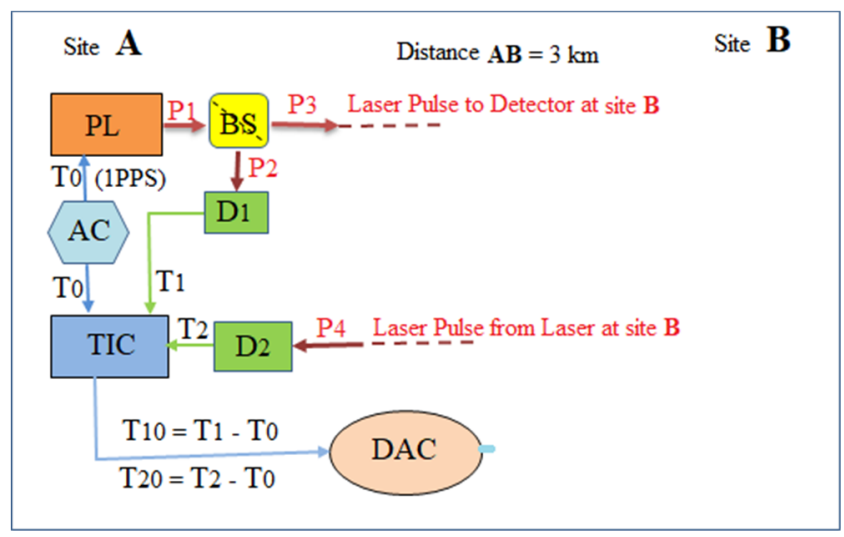

Figure 4. Schematic Connections and Signal Flow Diagram

PL: Picosecond DPSS Externally Triggered Pulsed Laser; BS: Beam Splitter.

AC: Cesium Beam Precision Atomic Clock; DAC: Data Acquisition Computer.

TIC: Time Interval Counter with 3 input channels and 1 Picosecond resolution.

$\mathrm{D}_{1}, \mathrm{D}_{2}$ : Ultrafast laser pulse InGaAs PIN photodetectors, UPDs or SPADs.

$\mathrm{P}_{1}, \mathrm{P}_{2}, \mathrm{P}_{3}, \mathrm{P}_{4}$ : Picoseconds Laser Pulses @ 1PPS.

$\mathrm{T}_{0}$ : 1PPS timing reference pulse to TIC and external trigger pulse to the Pulsed Laser.

$\mathrm{T}_{1}$ : Pulse $\mathrm{P}_{2}$ detection Timing Signal from detector $\mathrm{D}_{1}$.

$\mathrm{T}_{2}$ : Pulse $\mathrm{P}_{4}$ detection Timing Signal from detector $\mathrm{D}_{2}$.

$\mathrm{T}_{10}$ : Time Interval between timing signals $\mathrm{T}_{1}$ and $\mathrm{T}_{0}$, in Picoseconds, transferred to DAC.

$\mathrm{T}_{20}$ : Time Interval between timing signals $\mathrm{T}_{2}$ and $\mathrm{T}_{0}$, in Picoseconds, transferred to DAC. 


\section{Sequence of Operation}

To begin with 1PPS signal pulse $\left(\mathrm{T}_{0}\right)$ from the precision atomic clock (AC) triggers the pulsed laser (PL), while simultaneously another 1PPS pulse from AC is fed into the TIC as reference pulse $\mathrm{T}_{0}$. After a short characteristic time-delay, a picoseconds laser pulse $\mathrm{P}_{1}$ is emitted from the PL which passes through a beam-splitter (BS). In the $\mathrm{BS}$, the laser pulse $\mathrm{P}_{1}$ is split into two, $\mathrm{P}_{2}$ and $\mathrm{P}_{3}$. While the pulse $\mathrm{P}_{2}$ is fed into the nearby photodetector $\mathrm{D}_{1}$ through an optical fiber connector, the pulse $\mathrm{P}_{3}$ continues its journey through free space (air) in the original direction towards a photodetector at site $\mathrm{B}$. On detection of pulse $\mathrm{P}_{2}$ in photodetector $\mathrm{D}_{1}$, a Timing signal pulse $T_{1}$ is sent from $D_{1}$ to TIC. Next, the laser pulse $P_{4}$ coming from the laser at site $B$ gets captured in photodetector $\mathrm{D}_{2}$ and on detection $\mathrm{D}_{2}$ generates a Timing signal pulse $\mathrm{T}_{2}$ for feeding into TIC. After receiving the $\mathrm{T}_{2}$ signal input, the TIC computes two time-intervals, $\mathrm{T}_{10}\left(=\mathrm{T}_{1}-\mathrm{T}_{0}\right)$ and $\mathrm{T}_{20}\left(=\mathrm{T}_{2}-\mathrm{T}_{0}\right)$ and transfers them to the Data Acquisition Computer (DAC) for storage and further processing. After transferring the time interval data to the DAC, the TIC reverts back to the 'ready' state to receive the next 1 PPS or $T_{0}$ reference pulse.

\section{Following products from alternative sources can be considered for use in this experiment.}

A. Picosecond DPSS Pulsed Laser

(a) From PicoQuant, Germany. https://www.picoquant.com/ Laser Driver - Taiko PDL M1 with Laser Module - VisUV-532 nm

(b) From ALPHALAS GmbH, Germany. http://www.alphalas.com/ ALPHALAS PICOPOWER-LD Series with Driver and $670 \mathrm{~nm}$ wavelength.

(c) From AUREA Technology, France. http://www.aureatechnology.com/en/ Picosecond Pulsed Laser PIXEA - 665 nm

B. Three-channel Picosecond Event Timer

(a) From PicoQuant, Germany. https://www.picoquant.com/ HydraHarp 400 TIC (in TTTR mode only) with 4 channels and 1 ps resolution.

(b) From BNC, France. https://www.bncfrance.com/ T4100U TIC with 2 channels and 1 ps time resolution.

(c) From Carmel Instruments, USA. http://www.carmelinst.com/ NK732 TIC with 2 channels and 2 ps time resolution.

C. Ultrafast Photo Detector

(a) From ALPHALAS GmbH, Germany. http://www.alphalas.com/ UPD-30-VSG-P Ultrafast GaAs PIN Photodetectors - for $670 \mathrm{~nm}$ wavelength and Broadband Pulse Amplifier BBA-100.

(b) From AUREA Technology, France. http://www.aureatechnology.com/en/ LynXéa_VIS All-in-One 2-channel TCSPC module $400 \mathrm{~nm}$ to $1060 \mathrm{~nm}$.

(c) From HĀMAMATSU Photonics Japan. https://www.hamamatsu.com/ UltraFast MSM PhotoDetectors G4176-03 (GaAs) $500 \mathrm{~nm}$ to $850 \mathrm{~nm}$.

D. Cesium Atomic Clocks

(a) Microsemi's Cesium Clock- 5071A. https://tinyurl.com/y89v2hbj

(b) Oscilloquartz's Cesium Clock- OSA 3235B. https://tinyurl.com/ybrxnaag

\section{Data Analysis}

Since 1PPS reference and trigger pulses $\left(\mathrm{T}_{0}\right)$ are sent out from precision atomic clock regularly at one second interval, the computed time intervals $\mathrm{T}_{10}$ and $\mathrm{T}_{20}$ from the TIC at site A may be sequentially numbered with the consecutive number of 1 PPS pulses as $\mathrm{AT}_{10}(1), \mathrm{AT}_{10}(2), \ldots, \mathrm{AT}_{10}(\mathrm{n})$ and $\mathrm{AT}_{20}(1), \mathrm{AT}_{20}(2), \ldots, \mathrm{AT}_{20}(\mathrm{n})$. Similarly, the computed time intervals $\mathrm{T}_{10}$ and $\mathrm{T}_{20}$ from the TIC at site $\mathrm{B}$ may be sequentially numbered with the consecutive number of $1 \mathrm{PPS}$ pulses as $\mathrm{BT}_{10}(1), \mathrm{BT}_{10}(2), \ldots, \mathrm{BT}_{10}(\mathrm{n})$ and $\mathrm{BT}_{20}(1), \mathrm{BT}_{20}(2), \ldots, \mathrm{BT}_{20}(\mathrm{n})$. Finally, the data vectors $\mathbf{A T}_{\mathbf{1 0}}, \mathbf{A T}_{\mathbf{2 0}}, \mathbf{B T}_{\mathbf{1 0}}$, and $\mathbf{B T}_{\mathbf{2 0}}$ will be analyzed and processed with user software in the Data Acquisition Computer to establish the anisotropy of light speed $\mathrm{c}$ in the ECI reference frame.

\section{Copyrights}

Copyright for this article is retained by the author(s), with first publication rights granted to the journal.

This is an open-access article distributed under the terms and conditions of the Creative Commons Attribution license (http://creativecommons.org/licenses/by/4.0/). 